\title{
Bit-Interleaved Coded Modulation for the Relay Channel Using Bilayer LDPC Codes
}

\author{
Peyman Razaghi, Marko Aleksic and Wei Yu \\ Department of Electrical and Computer Engineering, \\ University of Toronto, Canada \\ e-mails: \{peyman,aleksicm,weiyu $\} @$ comm.utoronto.ca
}

\begin{abstract}
This paper describes a coding scheme for approaching the maximum theoretical decode-and-forward rate for the relay channel in the high signal-to-noise ratio (SNR) regime. The proposed scheme is based on a concatenation of bit-interleaved coded modulation (BICM) and a specially designed bilayer lowdensity parity-check (BLDPC) code that works simultaneously at two different SNRs. It is shown that the proposed BICMBLDPC scheme provides a close-to-optimal performance for the relay channel at high $\mathrm{SNRs}$, with a gap of $0.5 \mathrm{~dB}$ to the theoretical limit, at a bit error rate of $10^{-4}$.
\end{abstract}

\section{INTRODUCTION}

The relay channel is a three-terminal network in which a source terminal communicates with a destination terminal in the presence of a relay terminal that has no message of its own. A well-known relaying scheme is the decode-andforward (DF) approach [1, Theorem 1]. In the DF scheme, the relay decodes the source message and forwards a bin index for it to the destination. Recently, several practical low-SNR coding schemes based on turbo codes and low-density paritycheck (LDPC) codes have been proposed for implementing DF, e.g., [2], [3], [4], [5].

The main contribution of this paper is to devise a highSNR decode-and-forward scheme via a combination of bitinterleaved coded modulation (BICM) [6] and a class of specially designed LDPC codes called bilayer LDPC (BLDPC) codes [3]. In this scheme, the source terminal encodes its information bits using a conventional LDPC code; the binary LDPC source codeword is interleaved and mapped to a sequence of quadrature amplitude modulation (QAM) constellation symbols using Gray labeling. The relay decodes the source binary codeword, and generates extra parity bits for the source codeword. The extra parity bits, represented graphically by check nodes, modify the source LDPC graph by adding a second layer of check nodes to it, forming a bilayer LDPC code. These extra parity bits are encoded by the relay via a separate code, and transmitted to the destination. Finally, the destination first decodes the relay-generated parity bits, and then, decodes the source codeword over the bilayer LDPC graph. This paper demonstrates that in the high SNR regime, this BLDPC-BICM scheme can approach to the theoretical DF rate, within a gap of $0.5 \mathrm{~dB}$, at a bit error rate of $10^{-4}$.

The idea of using BICM and LDPC codes for DF is also considered in [4]. The approach of [4] is to adapt space-time coding schemes for the half-duplex relay channel. In [4], a conventional LDPC codeword of length $n$ is split into two

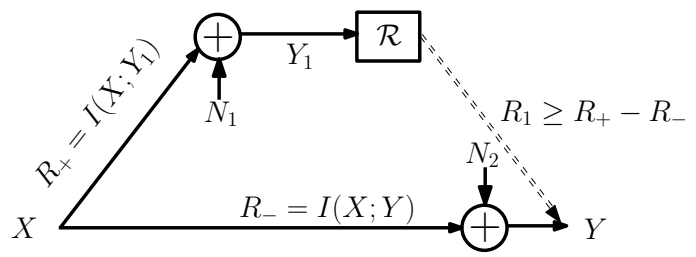

Fig. 1. A relay channel with a digital relay-destination link of rate $R_{1}$.

halves of length $n / 2$ each. The first half is transmitted by the source. The relay, upon decoding the source codeword based on the first half, transmits the second half, resembling a second antenna. Such an approach limits the rate of the LDPC code used at the source to be less than $1 / 2$, since the relay needs to decode the source message based on the first half of the source codeword. Our scheme resolves this limitation by using bilayer LDPC codes. Moreover, the proposed scheme is specifically designed so that all three source-relay, source-destination, and relay-destination links are utilized optimally.

\section{The Relay Channel with Digital RELAY-DESTINATION LINK}

The decode-and-forward scheme for the relay channel can be visualized by the simplified model shown in Fig. 1, where the relay-destination channel is a digital link with rate $R_{1}$. Let $X$ denote the source signal, $Y_{1}$ denote the received signal at the relay, and $Y$ denote the received version of the source signal at the destination. The channel inputs and outputs are related via $Y_{1}=X+N_{1}$, and $Y=X+N_{2}$, where $N_{1}$ and $\mathrm{N}_{2}$ represent circularly-symmetric complex Gaussian noises with zero mean, and variances $\sigma_{1}^{2} / 2$ and $\sigma_{2}^{2} / 2$ per dimension, respectively. The source-relay and source-destination SNRs are respectively defined by $\mathrm{SNR}_{+}=\frac{P}{\sigma_{1}^{2}}$ and $\mathrm{SNR}_{-}=\frac{P}{\sigma_{2}^{2}}$, where $P$ denotes the maximum source power. For this channel, the source-relay rate is defined by $R_{+}=I\left(X ; Y_{1}\right)$; the direct rate from the source to the destination is defined by $R_{-}=I(X ; Y)$. In addition, the relay can communicate to the destination via a digital link of rate $R_{1}$. Such a relay channel is obtained, for example, by digitizing the relay-destination channel in the frequency-division relay channel, or from the general relay channel [1], using a DF protocol.

The DF scheme achieves an overall source-destination rate $R_{+}$provided that $R_{1} \geq R_{+}-R_{-}$[1, Theorem 1]. This is achieved by a binning scheme summarized as follows. The 
relay decodes the source message of rate $R_{+}$, and forwards a bin index for the source message of rate $R_{1}$ to the destination. The bin index enables the destination to decode the original source message by restricting the source message to a bin of size $R_{+}-R_{1} \leq R_{-}$.

In a linear coding paradigm, a bin index for a codeword can be generated by forming extra parity bits, e.g., [7]. Using this idea, the above scheme translates into the following: the source encodes $k$ information bits in a codeword of length $n$ using a linear $(n, k)$ source code. Upon decoding the source codeword, the relay generates extra $r$ parities, and transmits them to the destination. Destination effectively observes a code of length $n$ with $n-k+r$ parities. With the extra parity bits, the destination is then able to decode the source codeword.

In order to achieve the theoretical DF rate using the above scheme, the source codebook should be capacity achieving at two rates, $R_{+}=k / n$, and $R_{-}=(k-r) / n$. Bilayer LDPC codes are specifically designed for this purpose for the low SNR regime [3]. The rest of the paper focuses on implementing the above parity-forwarding method at high SNR.

\section{Uncoded DF Using Set PARTItioning}

As a starting point, let us consider a simple uncoded DF scheme for high SNR. As outlined in the previous section, the role of the relay is to provide a bin index that reduces the number of candidate source messages to those inside a bin. A simple approach to implement binning is to partition the source constellation into several subconstellations. In such a scheme, the relay decodes the source transmitted symbol, and forwards the label of the subconstellation to which the source symbol belongs.

For example, consider a 16-QAM constellation used by the source (Fig. 2). We assume that $R_{1}=2$, i.e., the relay can communicate 2 bits per symbol to the destination. A natural partitioning of the source constellation is the set partitioning (SP), which divides the 16 constellation points into four subconstellations, each containing four symbols as shown in Fig. 2. In this case, each symbol is labeled by four bits where the last two bits correspond to the subconstellation label, and the first two bits identify the symbol within the subconstellation. For uncoded transmission, every four bits of the source information bit-sequence are mapped to one of the 16 constellation points. In each time slot, the relay decodes the source transmitted symbol (assuming that the source-relay channel is strong enough to provide a reasonably low BER). Upon decoding the source symbol, the relay re-transmits the last two bits of the source data to the destination via the digital link; e.g., if the source transmits the constellation point labeled by $(00,01)$, the relay transmits 01 .

The destination first decodes the relay-transmitted bits. The two bits transmitted by the relay restricts the source symbol to a four-point subconstellation of the original 16QAM constellation (e.g. the black-filled symbols in Fig. 2). The labeling of the original 16-QAM constellation is designed such that the minimum distance of the QPSK subconstellation $d_{2}$ is twice the minimum distance of the original 16-QAM constellation $d_{1}$, providing a coding gain of $6 \mathrm{~dB}$.

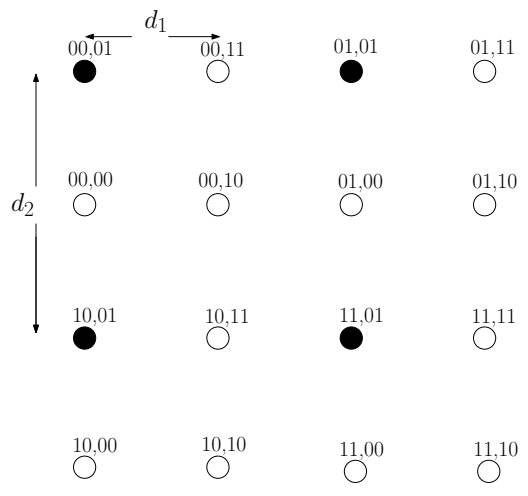

Fig. 2. Constellation set partitioning for Uncoded high SNR DF scheme. Note that the symbols in middle two columns are not Gray labeled.

In an uncoded transmission scenario, ideally all subconstellations and the mother constellation should be Gray labeled, in order to minimize the bit-wise errors at the relay and at the destination. However, due to the geometry of QAM constellation, this is not viable, resulting in higher bit-wise errors either at the relay or at the destination.

For coded system, the above set partitioning (SP) method can be incorporated in a trellis coded modulation (TCM) scheme in order to resolve the labeling problem and obtain a better performance. However, such a TCM-SP scheme for the relay channel requires nested convolutional codes, especially designed for this purpose. Conventionally, BICM is a simple alternative to coded modulation with a close-to-optimal performance at high SNRs. However, combining BICM and this SP scheme incurs a substantial loss, because Gray labeling is not viable for all subconstellations and the mother constellation, simultaneously. This problem can be resolved by using bilayer LDPC codes. The next section describes this BICM-BLDPC DF scheme.

\section{HIGH-SNR DF USING BICM AND BILAYER LDPC CODES}

A closer look at the previous approach shows that in the set partitioning method, binning is performed for each source symbol, rather than the entire source codeword. A more effective approach is to bin a sequence of source symbols, i.e., performing the binning over a larger block length. For linear codes, binning can be performed via the generation of extra parity bits, i.e., cosets play the role of bins. For LDPC codes, generating extra parity bits for a codeword corresponds to adding a second layer to the LDPC graph, resulting in a Bilayer LDPC code. To obtain a high-SNR DF scheme, bilayer LDPC codes can be concatenated with the BICM scheme. This section first provides a brief overview of BLDPC codes, then describes the BICM-BLDPC scheme.

\section{A. Bilayer LDPC codes}

A conventional LDPC code is a linear code defined over a bipartite graph consisting of variable nodes and check nodes. Check nodes represent linear constraints over the variable nodes. A variable node is connected to a check node via 


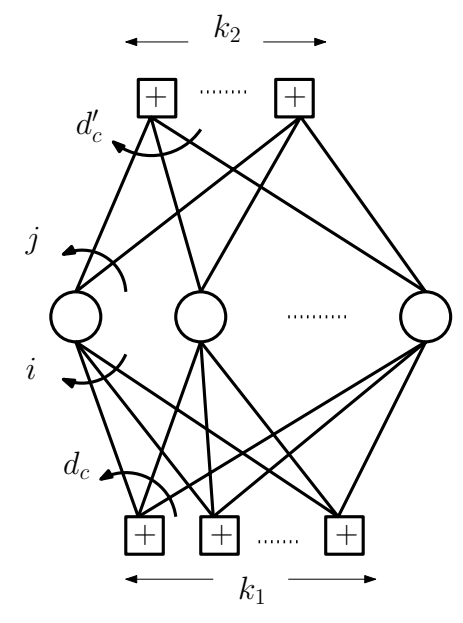

Fig. 3. Bilayer LDPC code graph.

an edge. The reader is kindly referred to [8] for a detailed characterization of LDPC codes.

In a bilayer LDPC code, a second (upper) layer is added to a base (lower) LDPC graph by adding extra parity-check nodes to the graph (Fig. 3). Each extra check node imposes an extra constraint, and is added to the graph by connecting a randomly selected set of variable nodes to it. The module-two sum of the selected variable nodes is assigned as the value of the new check node. Hence, a variable node may be connected to two types of edges: the lower edges, connecting a variable node to check nodes in the lower graph (lower check nodes); and the upper edges, connecting a variable node to upper check nodes. Thus, the degree of a variable node can be defined by the binary tuple $(i, j)$ if the variable node is connected to $i$ lower edges and $j$ upper edges. The degree distribution of a bilayer code determines the percentage of variable and check nodes of various degrees in the code graph.

Using a BLDPC code, a low-SNR DF scheme can be devised to approach the theoretical DF rate. In such a scheme, the source encodes its data using the lower layer of the BLDPC graph. The relay decodes the source codeword, and computes extra parity bits for the source codeword according to second layer. These extra parity bits constrain the source codeword to a coset, which is of a smaller size. (In other words, the destination effectively observes a source code with a lower rate.) The destination decodes the source codeword by message passing over the entire bilayer graph.

For this scheme to work, the degree distribution of a bilayer LDPC code should be optimized so that the lower layer code is capacity approaching at a higher SNR (at the relay), and the overall bilayer graph is capacity approaching at a lower SNR (at the destination). To optimize the degree distribution, a modified version of density evolution should be used to analyze the performance of a given bilayer code. To this end, bilayer density evolution is introduced in [3], which is used to determine the minimum SNRs at which the bilayer code can be decoded at the relay and the destination. Using bilayer density evolution, the degree distribution of the code can be optimized using an optimization procedure based on iterative linear programming [3]. Optimized BLDPC codes can be used to devise a high-SNR relaying scheme using BICM, as described in the next section.

\section{B. BICM-BLDPC High SNR DF}

In a BICM system [6], the output codeword of a binary encoder is interleaved; the interleaved sequence is then mapped to a sequence of constellation symbols via a labeling scheme. The resulting symbol sequence is modulated and transmitted over the channel. The decoding of the BICM codeword is based on computing the log-likelihood ratio (LLR) for every bit, given the received symbol sequence. (Details can be found in [6].) The performance of a BICM system depends on the particular labeling of the constellation points. It is conjectured that Gray labeling (in which the labels of every pair of adjacent constellation symbols differ only in one bit) gives the best performance for BICM [6].

The complete system is described in details in Fig. 4. The source encodes its binary information sequence using a conventional LDPC code. The binary codeword is mapped to a symbol sequence via an interleaver using Gray labeling. The relay decodes the source message by computing the LLRs associated with each bit position, and using the message passing algorithm. Upon decoding the source message, the relay generates extra parity parities for the source codeword (which adds a second layer to the source LDPC graph). The relay-generated parities are encoded using a separate code and transmitted to the destination. The destination first decodes the relay's message, obtaining extra parity constraints for the source codeword. Finally, the destination computes the LLRs for the source binary codeword from its received symbol sequence, and performs the decoding process over the bilayer LDPC graph using message passing.

\section{Simulation}

In this section, the performance of the proposed method is simulated for a pair of source-relay and source-destination rates. Ideally, the bilayer LDPC code component should be optimized for the effective bit channels between the source and the relay and between the source and the destination. However, as it will be demonstrated shortly, a BLDPC code, designed for a low-SNR binary-input relay channel with additive-white Gaussian noise (AWGN), performs close to the theoretical DF rate of the BICM system. We select the bilayer LPDC Code (A) designed for binary-input AWGN relay channel in [3]. For this code, the rates of the binary codes are $R_{-}^{b}=$ 0.6363 and $R_{+}^{b}=0.7000$, corresponding to the two layers ${ }^{1}$. Using quadrature amplitude modulator (QAM) with a 16 point constellation, the corresponding symbol rates are given by $R_{-}=4 R_{-}^{b}=2.5452$ and $R_{+}=4 R_{+}^{b}=2.8$.

Fig. 5 shows the bit error rate (BER) performance of this system at the relay and the destination, assuming a perfect channel of rate $R_{+}-R_{-}=0.2548$ from the relay to the destination. The minimum SNR to achieve a rate of $R_{-}=$

\footnotetext{
${ }^{1}$ Code (A) has a so-called bilayer expurgated structure, which is suitable when the difference between $R_{+}$and $R_{-}$is small. When the difference between $R_{+}$and $R_{-}$is large, an alternative bilayer lengthened LDPC code gives a better performance; see [3].
} 


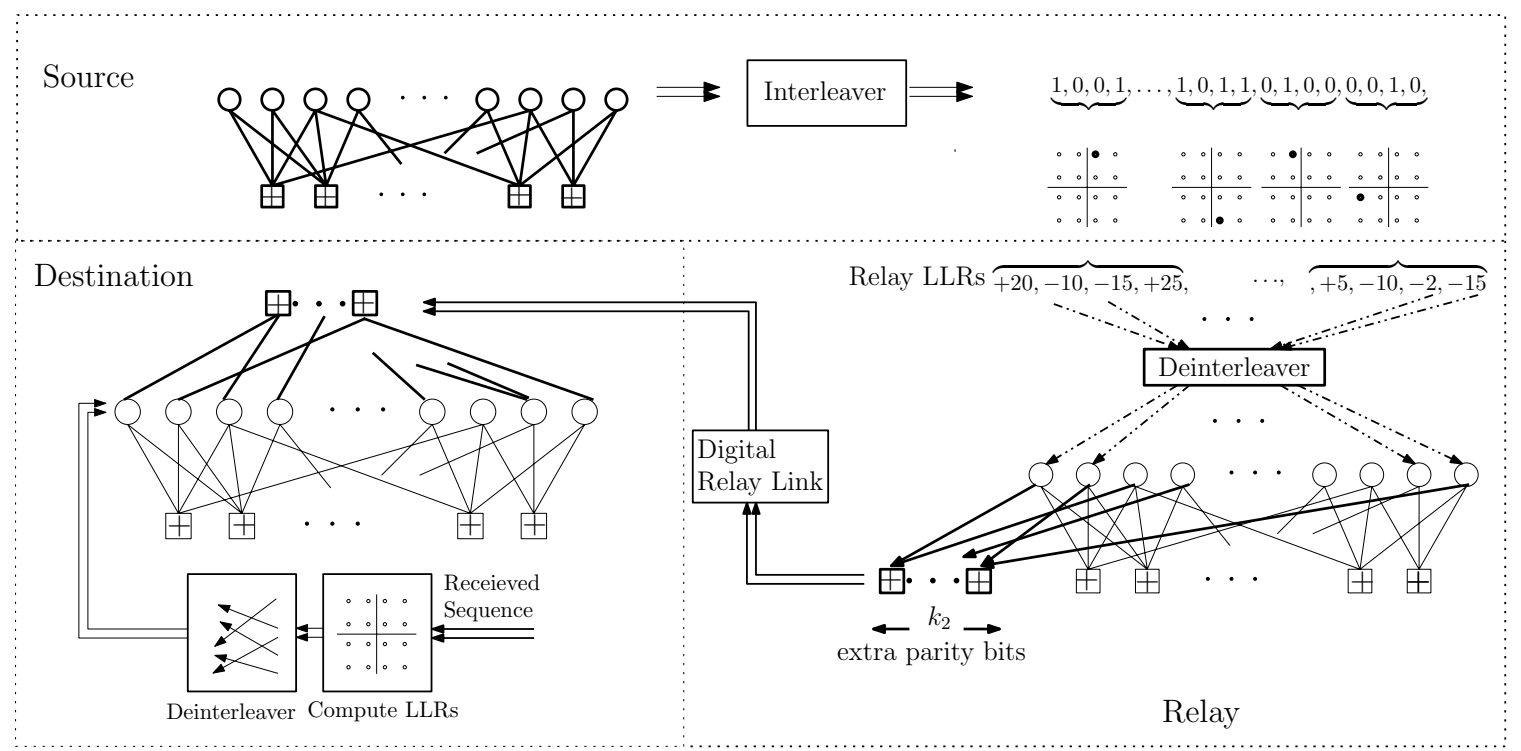

Fig. 4. High rate DF encoding based on concatenation of BICM and bilayer LDPC codes.

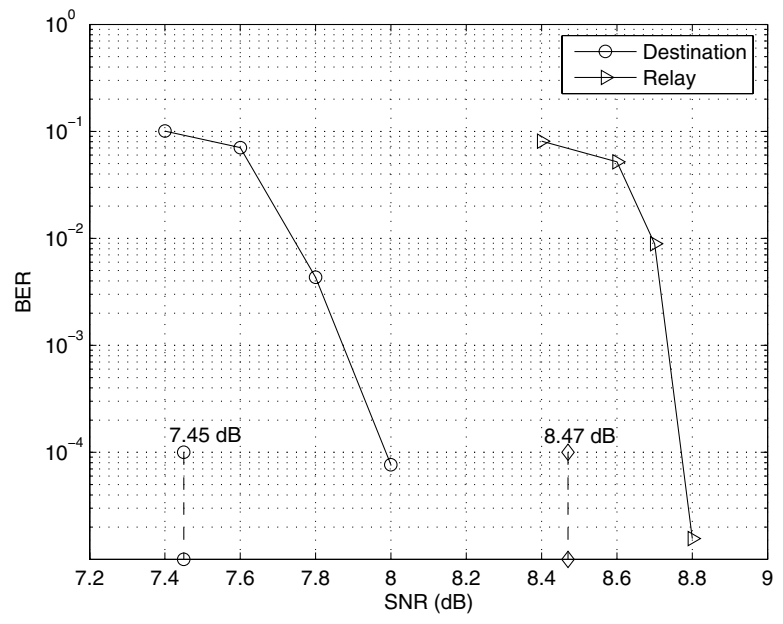

Fig. 5. Bit error rate performance of BICM-BLDPC code. The dashed lines represent the Shannon limits using 16-QAM source constellation.

2.5452 using the 16-QAM constellation is $\mathrm{SNR}_{-}=7.45 \mathrm{~dB}$. Similarly, the minimum SNR to achieve a rate of $R_{+}=2.8$, using 16-QAM, is $\mathrm{SNR}_{+}=8.47 \mathrm{~dB}$. According to the BER curves of Fig. 5 for a block length of 100,000, there is a gap of about $0.5 \mathrm{~dB}$ to the theoretical limit at a BER of $10^{-4}$ over both source-relay and source-destination channels ${ }^{2}$.

The performance of this scheme can be further improved by optimizing the bilayer graph for this specific channel, for example, by modifying the scheme of [9] for optimizing LDPC codes for BICM systems, and using the design procedure of [3]. However, the achieved gap to the theoretical limit, without optimizing the BLDPC code specifically for this BICM chan-

${ }^{2}$ However, we expect error floors to occur beyond $10^{-4}$. The effect of error floors can be alleviated for example by using a high rate outer code. It should also be noted that error propagation also deteriorates the overall performance of the system, whereby, an erroneous decoding of the source codeword occurs at the relay. nel, is already quite small $\left(0.5 \mathrm{~dB}\right.$ at $\left.10^{-4}\right)$, suggesting that the gain of optimizing the code for the BICM system would be marginal.

\section{CONCLUSION}

In this paper, we devised a DF encoding scheme for the relay channel using a combination of BICM and bilayer LDPC codes. The performance of the proposed scheme is simulated using a bilayer LDPC code, optimized for the binary-input AWGN. Similar to the single-user BICM scheme, it is shown that the proposed BICM-BLDPC scheme provides a close-tooptimal performance at high SNRs, with a gap of $0.5 \mathrm{~dB}$ at $10^{-4}$ to the theoretical limit.

\section{REFERENCES}

[1] T. M. Cover and A. A. El Gamal, "Capacity theorems for the relay channel," IEEE Trans. Inform. Theory, vol. 25, no. 5, pp. 572-584, Sept. 1979.

[2] Z. Zheng and T. M. Duman, "Capacity-approaching turbo coding and iterative decoding for relay channels," IEEE Trans. Commun., vol. 53, no. 11, pp. 1895 - 1905, Nov. 2005.

[3] P. Razaghi and W. Yu, "Bilayer low-denisty parity-check codes for decode-and-forward in relay channels," Submitted to IEEE Trans. Inf. Theory, 2006.

[4] G. Kramer, "Distributed and layered codes for relaying," in Conference Record of the Thirty-Ninth Asilomar Conference on Signals, Systems and Computers, Oct. 2005, pp. 1752- 1756.

[5] P. Razaghi and W. Yu, "Bilayer LDPC codes for the relay channel," in Proc. of IEEE Int. Conf. Commun. (ICC), June 2006, vol. 4, pp. 16781682.

[6] G., Caire, G., Taricco, E., and Biglieri, "Bit-interleaved coded modulation," IEEE Trans. Inform. Theory, vol. 44, no. 3, pp. 927-946, May 1998.

[7] R. Zamir, S. Shamai, and U. Erez, "Nested linear/lattice codes for structured multiterminal binning," IEEE Trans. Inform. Theory, vol. 48, no. 6 , pp. 1250-1276, June 2002.

[8] T. Richardson and R. Urbanke, "The capacity of low-density parity-check codes under message-passing decoding," IEEE Trans. Inform. Theory, vol. 2, pp. 599-618, Feb. 2001.

[9] M. Ardakani, F. R. Kschischang, and W. Yu, "Near-capacity coding for discrete multitone systems with impulse noise," EURASIP J. Applied Signal Processing, vol. 2006, pp. 1-10, Article ID 98738. 